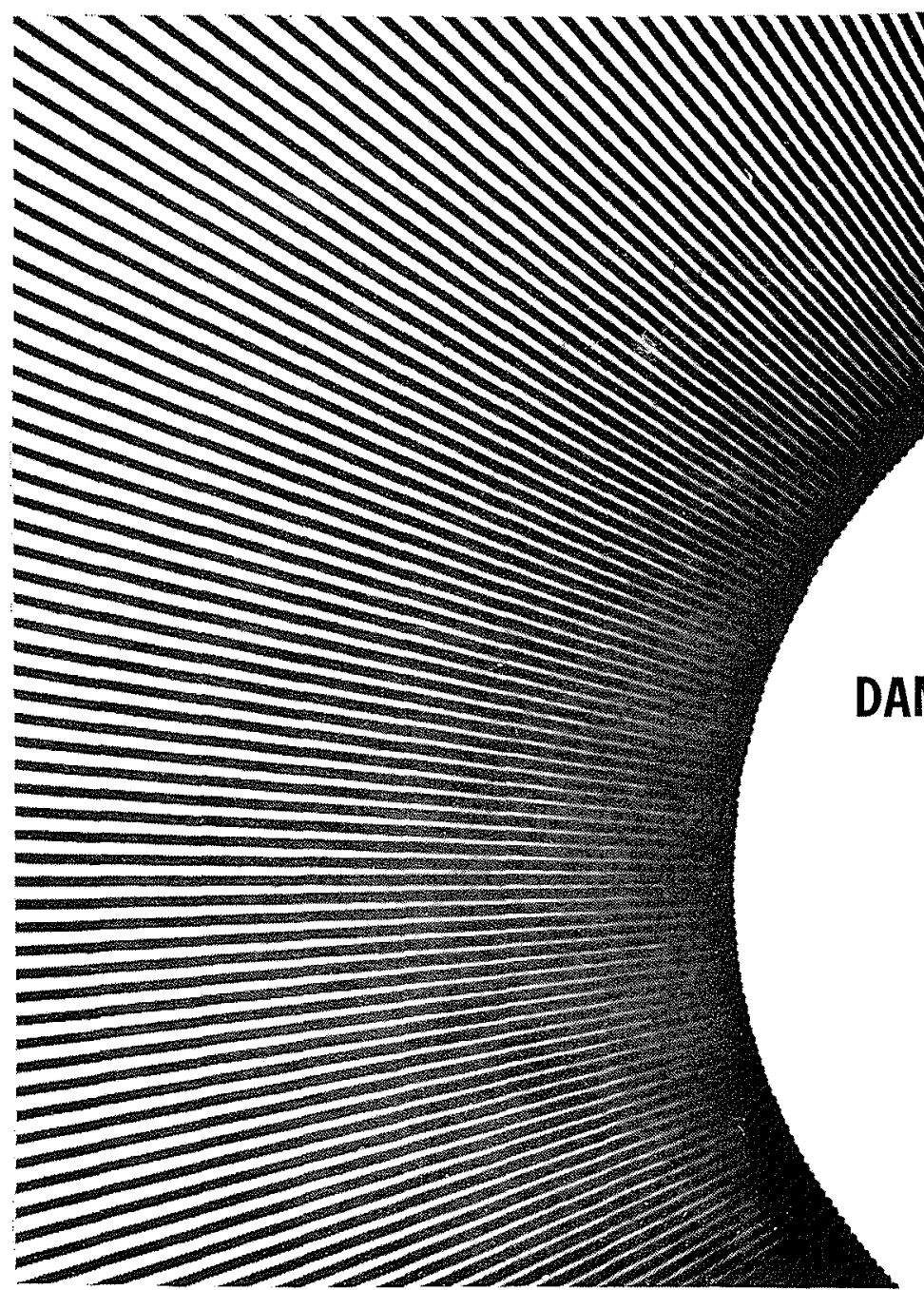

\title{
PRESSIONS ET VITESSES DANS UNE LAME DÉVERSANT SUR UN SEUIL EN MINCE PAROI
}

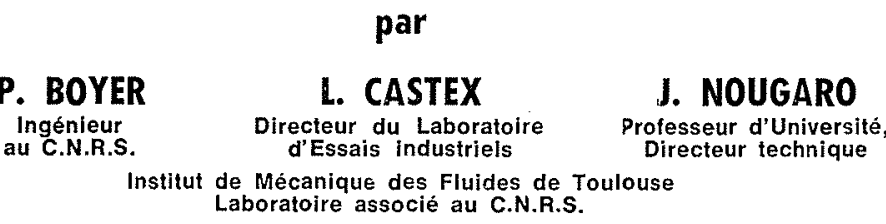

P. BOYER

Ingénieur. Institut de Mécanique des Fluides de Tou
Laboratoire associé au C.N.R.S.
Le déversement sur un seuil qui semble a priori bien connu, laisse subsister de nombreuses incertitudes dans la répartition hydrodynamique. C'est pourquoi nous avons pensé qu'une analyse des pressions dans la lame déversante au dessus du seuil pouvait contribuer à la connaissance du phénomène.

L'influence de l'arête du seuil crée une singularité, par suite une contraction dans la partie inférieure de l'écoulement. Il s'ensuit une discontinuité de la ligne de répartition des pressions statiques. Cet effet est prépondérant sur la vitesse des filets qui dans cette zone subissent une accélération très importante. Il semblait donc tout indiqué d'analyser les vitesses dans cette région, et par suite d'étendre cette recherche dans tout le plan vertical contenant le parement amont du seuil mince.

Afin de généraliser cette analyse nous avons multiplié les conditions en examinant les effets sur des canaux de plusieurs dimensions et des seuils de hauteurs différentes.

$1 /$

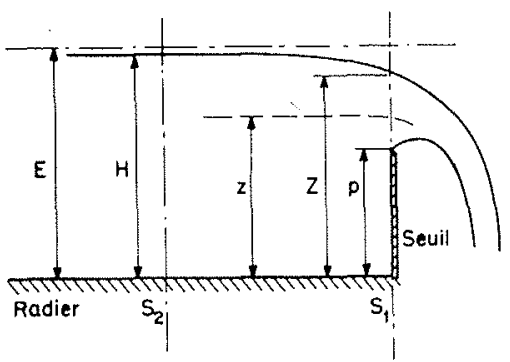

Les trois procédés de mesure mis en œuvre donnent des résultats assez semblables quels que soient les cas considérés.

Dès lors, il nous a été possible d'adopter une représentation regroupant tous les paramètres de l'écoulement, et le spécialiste, amené à calculer un déversoir voit sa tâche simplifiée. Il est bien évident que nous ne voulons pas annihiler les travaux antérieurs, mais au contraire, nous montrons que la répartition des pressions et des vitesses apporte dans ce domaine un élément nouveau.

Expériences réclisées

Notations.

Le nombre de paramètres influençant la lame justifie la figure 1 .

Le radier du canal étant considéré horizontal, son plan sera pris comme référence pour toutes les cotes.

Nous appellerons :

$\mathrm{E}$ : énergie interne du fluide rapporté à l'unité de poids, calculée en amont de la section $\mathrm{S}_{2}$;

$\mathrm{H}$ : tirant d'eau dans le canal au droit de la section $\mathrm{S}_{2}$; distance $\mathrm{S}_{1} \mathrm{~S}_{2}>5 \mathrm{P}$

$Z$ : tirant d'eau au-dessus du seuil dans la section $S_{1}$

$z$ : côte d'un filet liquide, on utilisera très souvent $(z-\mathrm{P})$ pour ramener ce filet à la crête du seuil; 
P: hauteur de pelle;

$\mathrm{S}_{1}$ : section droite de l'écoulement contenant le parement amont du seuil;

$\mathrm{S}_{2}$ : section droite de l'écoulement en amont du seuil défini en position ci-dessus;

B : largeur du canal;

$\mathrm{P}_{\text {I }}$ : pression totale mesurée par les sondes; valeur sensiblement égale à $\mathrm{E}$;

$\mathrm{P}_{\mathrm{s}}$ : pression statique du fluide par rapport au seuil;

$\mathrm{V}_{1}{ }^{2} / 2 \mathrm{~g}$ : énergie cinétique du fluide par unité de poids en $\mathrm{S}_{1}$

$\mathrm{V}_{2}^{2} / 2 \mathrm{~g}$ : énergie cinétique du fluide par unité de poids en $\mathrm{S}_{2}$;

$\mathrm{V}_{\tilde{x}^{2}}^{2 / 2} \mathrm{~g}$ : énergie cinétique d'un filet de cote $z$ dans le plan $S_{1}$

$\alpha$ : angle des filets dans le plan horizontal par rapport à l'axe du canal;

$\tau$ : angle des filets dans le plan vertical par rapport à l'horizontale;

$\mathscr{T}$ : somme de la pression de position par rapport à la crête et de la pression statique mesurée :

$$
\mathscr{T}=(\mathrm{P}-z)+(p / \rho g)
$$

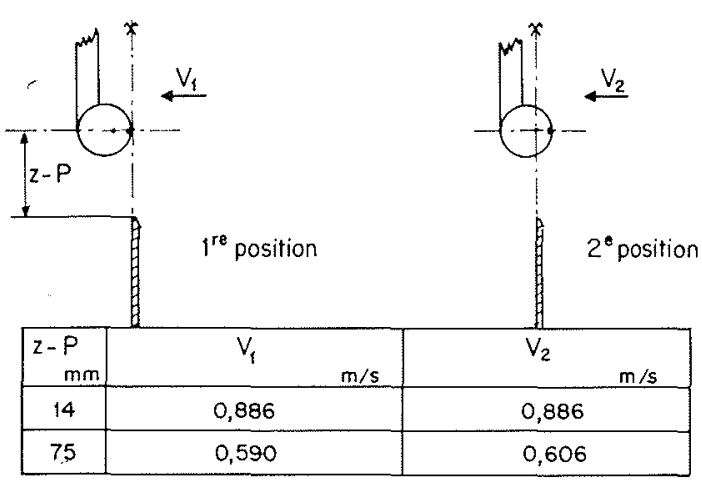

2/

\section{Domaine expérimental.}

Les expériences ont été conduites dans deux canaux distincts :

- le premier de largeur $B=0,30 \mathrm{~m}$;

- le second de largeur $B=0,10 \mathrm{~m}$.

Les déversoirs avaient une hauteur de pelle de $\mathbf{P}=0,30 \mathrm{~m}$ et $\mathbf{P}=0,40 \mathrm{~m}$ dans le canal $\mathrm{n}^{\circ} 1$ (série d'essais $I$ et $\mathrm{II}$ ) et $\mathrm{P}=0,20 \mathrm{~m}$ dans le canal $\mathrm{n}^{\circ} 2$ (série d'essais III, IV et V).

Les trois procédés expérimentaux permettant la comparaison des résultats sur les deux types d'installations se classent :

a) Sonde anémoclinométrique :

- essais série I : $\mathrm{P}=0,40 \mathrm{~m} ; \mathrm{B}=0,30 \mathrm{~m}$;

- essais série II : $\mathrm{P}=0,30 \mathrm{~m} ; \mathrm{B}=0,30 \mathrm{~m}$;

- essais série III : $P=0,20 \mathrm{~m} ; \mathrm{B}=0,10 \mathrm{~m}$.

b) Sonde de Prandtl:

- essai série IV : $\mathrm{P}=0,20 \mathrm{~m}, \mathrm{~B}=0,10 \mathrm{~m}$.

c) Méthode chronophotographique :

- essai série $V: P=0,20 \mathrm{~m} ; \mathrm{B}=0,10 \mathrm{~m}$.

A toutes ces conditions plusieurs charges ont été appliquées et le tableau suivant conne l'éventail des expériences.

\begin{tabular}{|c|c|c|c|c|c|}
\hline & $(\mathrm{H}-\mathrm{P})$ & $\underset{m}{(E-P)}$ & $(\mathrm{Z}-\mathrm{P})$ & $\begin{array}{c}Q \\
\mathrm{l} / \mathrm{s}\end{array}$ & $N *$ \\
\hline $\begin{array}{l}\text { Essai } \\
\text { série I }\end{array}$ & $\begin{array}{l}0,070 \\
0,090 \\
0,110 \\
0,130 \\
0,150 \\
0,170\end{array}$ & $\begin{array}{l}0,0703 \\
0,0905 \\
0,01109 \\
0,13142 \\
0,15202 \\
0,17281\end{array}$ & $\begin{array}{l}0,0603 \\
0,0779 \\
0,0950 \\
0,1122 \\
0,1282 \\
0,1462\end{array}$ & $\begin{array}{l}10,50 \\
15,20 \\
20,55 \\
26,55 \\
32,85 \\
40,20\end{array}$ & $\begin{array}{l}3 \\
3 \\
4 \\
5 \\
6 \\
7\end{array}$ \\
\hline $\begin{array}{c}\text { Essai } \\
\text { série II }\end{array}$ & $\begin{array}{l}0,0761 \\
0,0951 \\
0,1196 \\
0,1306 \\
0,1774 \\
0,2254 \\
0,2386\end{array}$ & $\begin{array}{l}0,0766 \\
0,0960 \\
0,1213 \\
0,1327 \\
0,1820 \\
0,2334 \\
0,2476\end{array}$ & $\begin{array}{l}0,0635 \\
0,0800 \\
0,1000 \\
0,1100 \\
0,150 \\
0,191 \\
0,203\end{array}$ & $\begin{array}{l}11,65 \\
16,20 \\
22,50 \\
26,90 \\
43,60 \\
63,25 \\
69\end{array}$ & $\begin{array}{l}4 \\
4 \\
4 \\
5 \\
6 \\
7 \\
7\end{array}$ \\
\hline $\begin{array}{c}\text { Essai } \\
\text { série III }\end{array}$ & 0,0973 & 0,0992 & 0,0855 & 5,815 & 9 \\
\hline $\begin{array}{c}\text { Essai } \\
\text { série IV }\end{array}$ & 0,0973 & 0,0992 & 0,0855 & 5,815 & 17 \\
\hline $\begin{array}{c}\text { Essai } \\
\text { série V }\end{array}$ & $\begin{array}{l}0,0838 \\
0,0973 \\
0,1198 \\
0,1288\end{array}$ & $\begin{array}{l}0,0851 \\
0,0992 \\
0,1230 \\
0,1325\end{array}$ & $\begin{array}{l}0,0742 \\
0,0855 \\
0,1058 \\
0,1148\end{array}$ & $\begin{array}{l}4,582 \\
5,815 \\
8,102 \\
8,919\end{array}$ & $\begin{array}{r}44 \\
163 \\
46 \\
58\end{array}$ \\
\hline
\end{tabular}

$\mathrm{N}^{*}$ : Nombre de points d'ordonnée $(z-\mathrm{P})$ pris en compte pour chaque condition d'écoulement.

Les mesures couvrent les limites habituelles proposées par Bazin et Rehbock.

\section{Description des installations.}

Les installations utilisées étaient rigoureusement identiques dans leur principe de fonctionnement. Les canaux étaient alimentés par des bassins d'accumulation à niveau constant. Les seuils implantés à l'extrémité des canaux permettaient d'avoir une lame à la pression atmosphérique. Latéralement la largeur du jet était maintenue constante.

Les seuils minces fabriqués en alliage léger $(5 \mathrm{~mm})$ répondaient aux normes de l'I.S.O. Des prises de pression percées dans le parement amont et suivant l'axe médian ont permis une mesure sur toute la hauteur de la pelle.

En aval, le flot était canalisé vers des déversoirs de mesure.

\section{Processus expérimentaux}

\section{Conduite des essais.}

Une sonde anémoclinométrique, déjà utilisée dans notre laboratoire à des travaux comparables, a été retenue comme première méthode pour mesurer les vitesses dans les essais I et II. L'inconvénient de l'appareil réside dans le fait que les profils de vitesse obtenus demeurent incomplets aux limites extrêmes de la lame. Pour une utilisation ration- 
nelle, la tête de mesure ne doit pas être approchée à moins de deux fois son diamètre d'une paroi ou de la surface libre. La courbe de répartition ne pouvant se raccorder ni à la crête ni à la surface libre, il était impossible de planimétrer l'aire des vitesses.

Nous étant proposé d'étudier d'une façon complète la valeur des vitesses dans toute la section de déversement, il ne pouvait subsister de zone inexplorée. Afin de déterminer ces vitesses à moins de $1 \mathrm{~mm}$ du seuil et de la surface libre une sonde de Prandtl de fabrication spéciale a donné un nouveau moyen d'investigation et de comparaison.

Ayant constaté un léger décalage des courbes obtenues par les deux procédés, il nous a paru intéressant de poursuivre ces expériences sur une nouvelle installation (essai IV) et d'y adapter un troisième procédé de mesure. La méthode chronophotographique a donc conclu cette recherche.

\section{Soncie sphérique.}

11 est inutile de rappeler le principe et les conditions d'utilisation de lappareil, un article inséré dans le Génie civil $n^{\circ} 4$ de novembre 1969 traite du procédé.

Une indication sur la position de l'instrument par rapport au plan que l'on souhaite explorer doit être précisée. Pour cela deux positions de la sonde ont été comparées comme l'indique la figure 2. Le premier cas est le plus logique puisque le point d'arrêt est compris dans le plan d'exploration. Dans le deuxième cas, ce sont les prises de pression latérales qui sont confondues avec le plan.

Les deux positions montrent une translation horizontale du point d'arrêt traduisant une différence de vitesse de $0 \%$ près du seuil à $3 \%$ près de la surface libre.

\section{Sonde de Prandt!.}

Pour une approche suffisante des limites de la lame et obtenir un profil complet, nous avons été amenés à fabriquer une sonde constituée par deux tubes d'acier inoxydable de diamètre extérieur $\varnothing=1,2 \mathrm{~mm}$ séparés par une distance horizontale de $6 \mathrm{~mm}$. Chaque antenne mesurait respectivement la pression totale et la pression statique pour une même cote $z-P$.

Le diamètre intérieur de ces tubes étant très faible $(0,6$ $\mathrm{mm}$ ) le temps de stabilisation de la colonne d'eau était atteint au bout de cinq minutes.

Tout comme le tube de Pitot l'emploi de ce type de sonde est limitatif. Il est nécessaire que sa direction soit très proche de la direction des filets liquides. Il a donc fallu déterminer l'incidence de ceux-ci dans toute l'épaisseur de la lame et superposer les sondes aux tangentes des trajectoires. Une photographie des filets, visualisés au moyen de poudre d'aluminium, a donné pour chaque point de cote $(z-P)$ l'angle des tangentes $\tau$ (fig. 3 ).

Pour obtenir une superposition correcte de la sonde avec les tangentes dans le plan de mesure, il nous a été indispensable de réaliser un appareil spécial articulé en deux points comme l'indique la figure 4.

\section{Méthode chronophotograpnique.}

L'écoulement étant considéré comme plan, le procédé chronophotographique était donc tout indiqué comme troisième moyen d'investigation.

Pour nos conditions expérimentales nous avons adopté : - un appareil photographique Nikon équipé d'un objectif de $200 \mathrm{~mm}$;

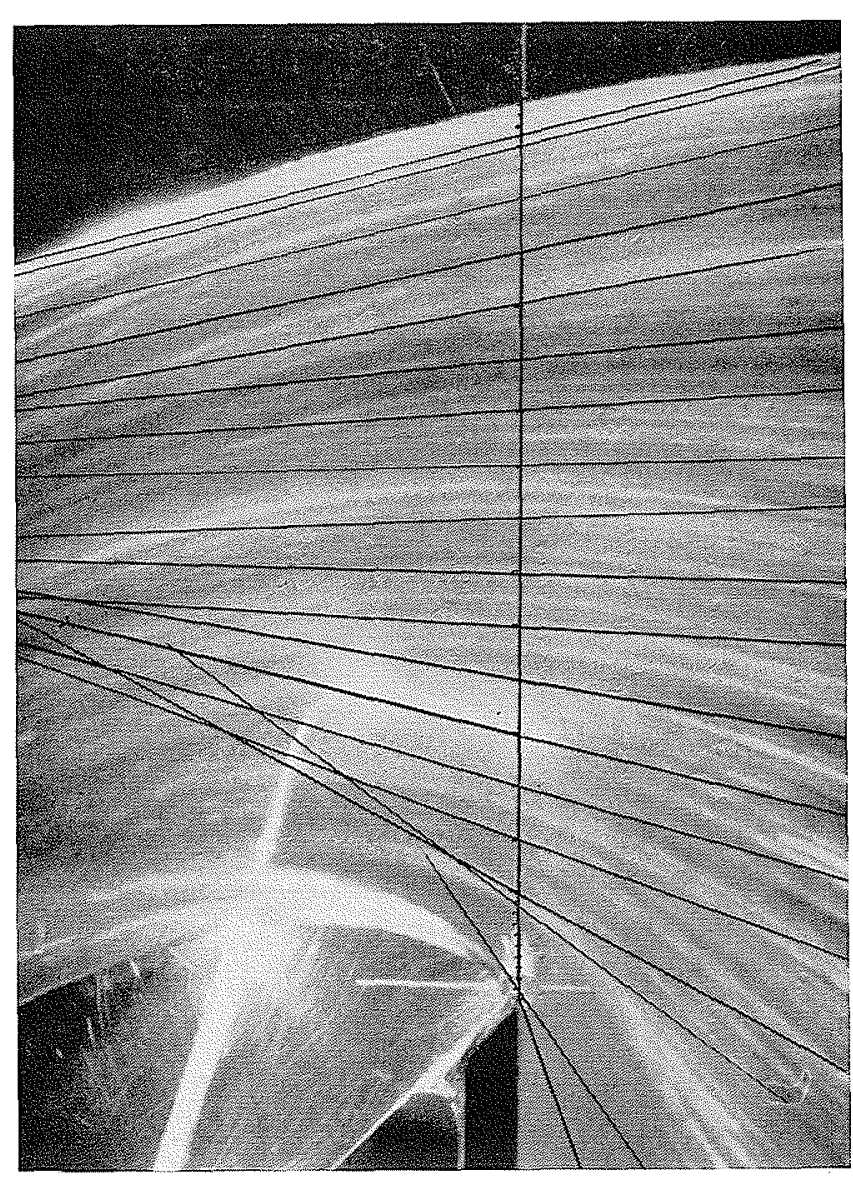

$3 /$

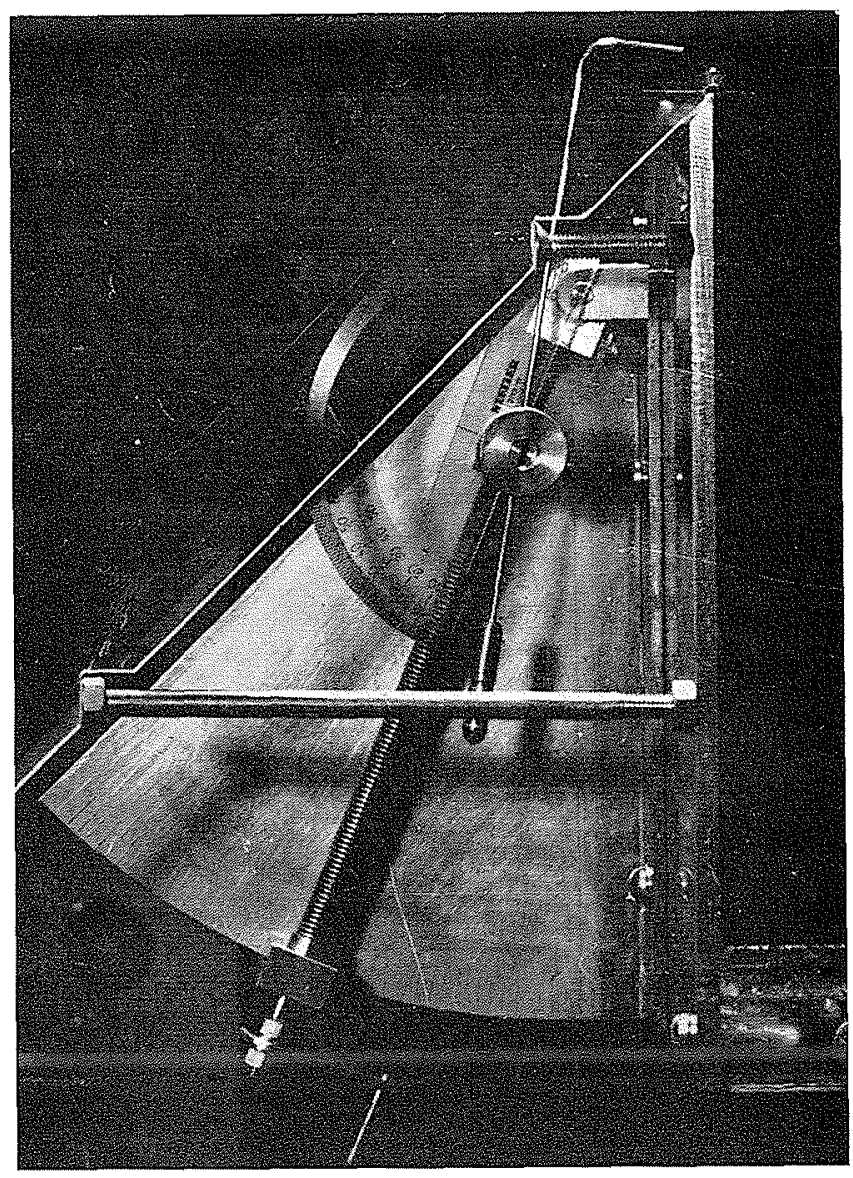

4/ 


\section{P. BOYER, L. CASTEX ef J. NOUGARO}

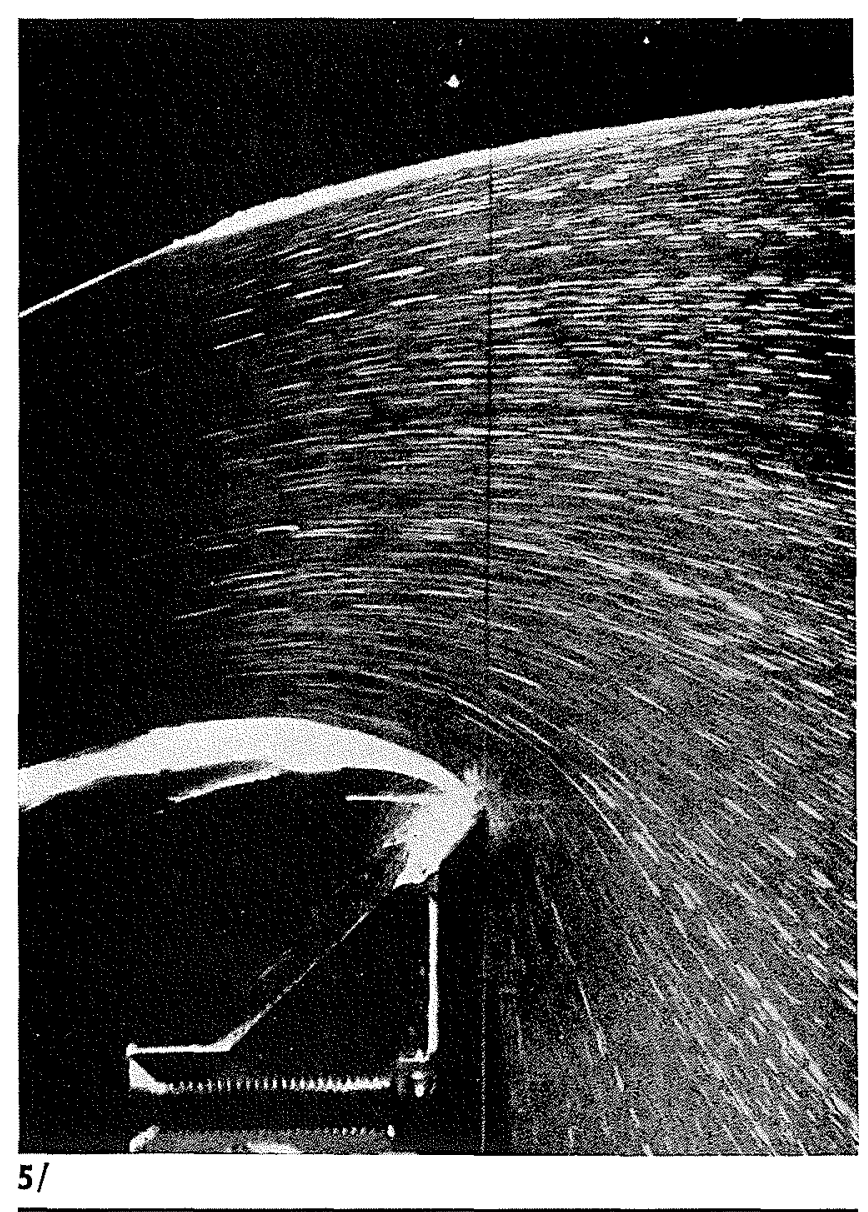

- un disque entaillé de quatre fentes entraîné par un moteur branché à une alimentation stabilisée.

La période d'exposition était comprise entre $7 / 1000$ et $2 / 100$ de secondes.

La figure $\mathrm{n}^{\circ} 5$ est l'un des vingt-cinq clichés exploités.

Le dépouillement a été réalisé par projection des négatifs sur un écran. La courbe moyenne des points obtenus est déduite de deux méthodes.
La première consiste à calculer un point par moyenne arithmétique sur d'égales tranches d'ordonnées. La seconde donne la courbe réelle par l'intermédiaire d'un calculateur.

\section{Etude des paramètres influencant le débit}

\section{Variation de l'angle $\alpha$.}

L'angle $\alpha$ est l'angle du vecteur vitesse dans le plan horizontal. Mesuré dans les essais I, II et III, il prend des valeurs maximales de 8 " près des parois, révélant les effets de bords et de rugosité. Dans l'axe de la lame l'écart maximal est de $\pm 1,5^{\circ} \mathrm{cc}$ qui permet d'admettre que l'écoulement est plan.

\section{Variation de l'angle $\tau$.}

L'angle $\tau$ est l'angle du vecteur vitesse dans le plan vertical. Il a été mesuré à la sonde anémoclinométrique pour les essais I, II et III et par visualisation pour les essais IV et $\mathrm{V}$. La variation de cet angle est donnée dans la figure 6. Afin de généraliser tous les cas, l'ordonnée est graduée en valeurs adimensionnelles de $(z-\mathrm{P}) /(\mathrm{Z}-\mathrm{P})$.

L'écart pratiquement constant entre les deux courbes et voisin de $7^{\circ}$ correspond aux moyens de mesure adoptés.

On peut remarquer que lorsque $(z-P) /(Z-P)$ tend vers $1,-14^{\circ}<\tau<-7^{\circ}$, les filets descendent. Lorsque $\tau=0$, on a :

$$
0,525<(z-\mathrm{P}) /(\mathrm{Z}-\mathrm{P})<0,675
$$

Enfin lorsque $\tau>50^{\circ},(z-\mathrm{P}) /(\mathrm{Z}-\mathrm{P})$ tend vers 0 . L'observation a montré que les filets passant au ras du seuil ont une direction ascendante presque verticale. Finalement nous pensons que la courbe des essais IV et V correspond aux incidences réelles. Il est regrettable que ce procédé ne soit applicable qu'à des expériences très limitées.

Toutefois nous souhaitons apporter la conviction que I'utilisation d'une sonde anémoclinométrique dans tous les autres cas demeure satisfaisante.

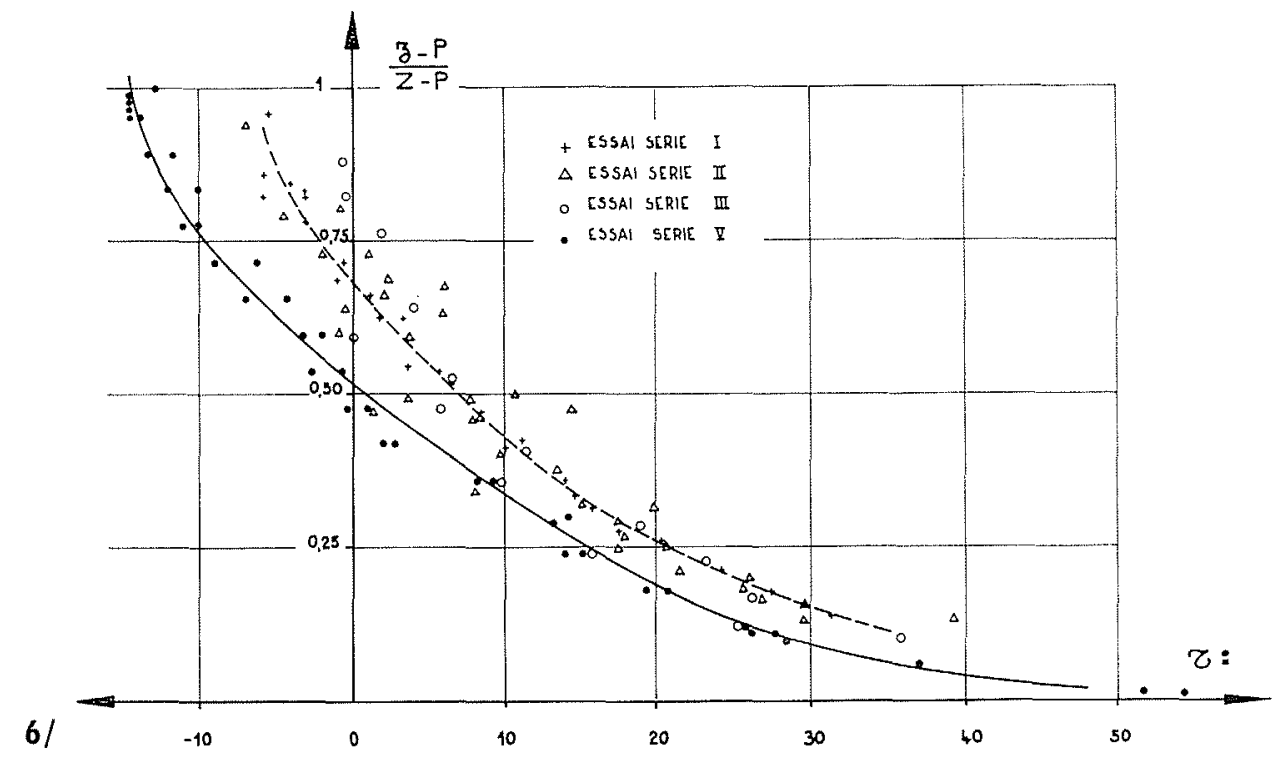


Etude des profils de vitesse.

Ceci représente le but initial de notre étude puisque l'incertitude subsistait tant sur la répartition que sur la valeur aux limites.

Dans une première phase, les essais III et IV ayant été effectués, leur comparaison pour une même condition d'écoulement permet de remarquer (fig. 7) :

- que lorientation des directrices diffère légèrement dans la partie supérieure;

- que d'une manière générale les vitesses obtenues par la sonde sont plus faibles que celles obtenues avec les antennes de Prandtl.

Dans une deuxième phase, afin de lever le doute sur ces résultats, l'essai $\mathrm{V}$ a donné un sens réel et déterminant.

Le dispositif chronophotographique étant réglé il a été facile de multiplier le nombre d'expériences. Bien que quatre charges sur le seuil aient servi à l'étude nous donnons dans la figure 8 , la comparaison pour une valeur de $z-\mathrm{P}$ des trois méthodes expérimentales.

La méthode chronophotographique conduit à un grand nombre de points (163) présentant une dispersion importante. Nous avons pu obtenir une première courbe de répartition (1) par moyenne arithmétique. La deuxième courbe (2) programmée sur le principe du développement polynomial de Tchébychev a pour équation :

$$
\begin{aligned}
& (z-P) /(Z-P)=-46 V_{1}{ }^{4}+150 V_{1}{ }^{3} \\
& -175 V_{1}{ }^{2}+84 V_{1}-13
\end{aligned}
$$

La superposition des courbes (3) essai III et (4) essai IV sur la figure 8 indique que :

- les courbes moyennes et lissées sont en bonne concordance;

- les vitesses aux limites sont déterminées;

- les courbes (2) et (3) sont pratiquement confondues;

- entre les courbes (2) et (4) on note un écart de $8 \%$ à la surface libre et $15 \%$ au voisinage du seuil.

REMARQUE: Les recherches bibliographiques nous ont montré que Bazin avait déjà étudié ce problème. Mais le dispositif expérimental assez rudimentaire et les surfaces étudiées étant légèrement différentes font que les résultats divergent un peu.

Bazin propose :

- vitesse à la surface libre:

$$
\mathrm{V}_{1}^{\prime}=0,475 \sqrt{2 g(\mathrm{H}-\overline{\mathrm{P}})}
$$

- vitesse auprès du seuil :

soit :

$$
\mathrm{V}^{\prime \prime}{ }_{1}=0,946 \sqrt{2 g(\mathrm{H}-\mathrm{P})}
$$

$$
V^{\prime \prime}{ }_{1} / V^{\prime}{ }_{1}=2
$$

Nous avons obtenu:

$$
\begin{gathered}
\mathrm{V}^{\prime}=0,412 \sqrt{2 g(\mathrm{H}-\mathrm{P})} \\
\mathrm{V}^{\prime \prime}=1,010 \sqrt{2 g(\mathrm{H}-\mathrm{P})} \\
\mathrm{V}^{\prime \prime} / \mathrm{V}^{\prime}=2,45
\end{gathered}
$$

Comparaison des débits mesurés avec les débits déduits de l'intégration de vitesses.

Pour obtenir un débit à partir de l'intégration du profil des vitesses, il est nécessaire de considérer la composante

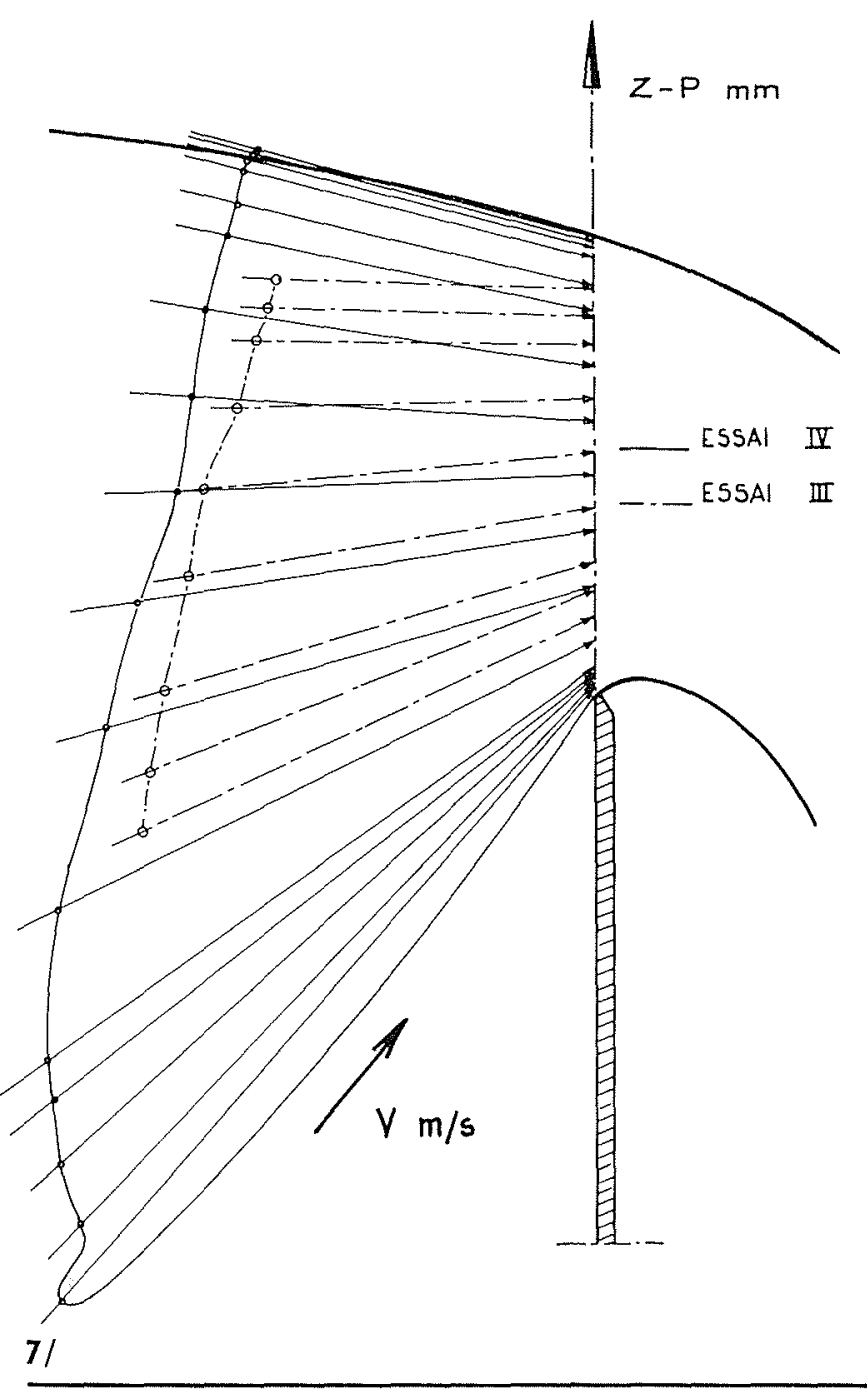

normale des vecteurs par rapport au plan de déversement. Soit $\vec{V} \cos \tau$.

Une première comparaison pour les essais $\mathrm{I}$, II et III pour lesquels les vitesses étaient mesurées au moyen de sondes est donnée par la figure 9. L'écart ne dépasse pas $3 \%$.

Une deuxième comparaison pour un même tirant d'eau

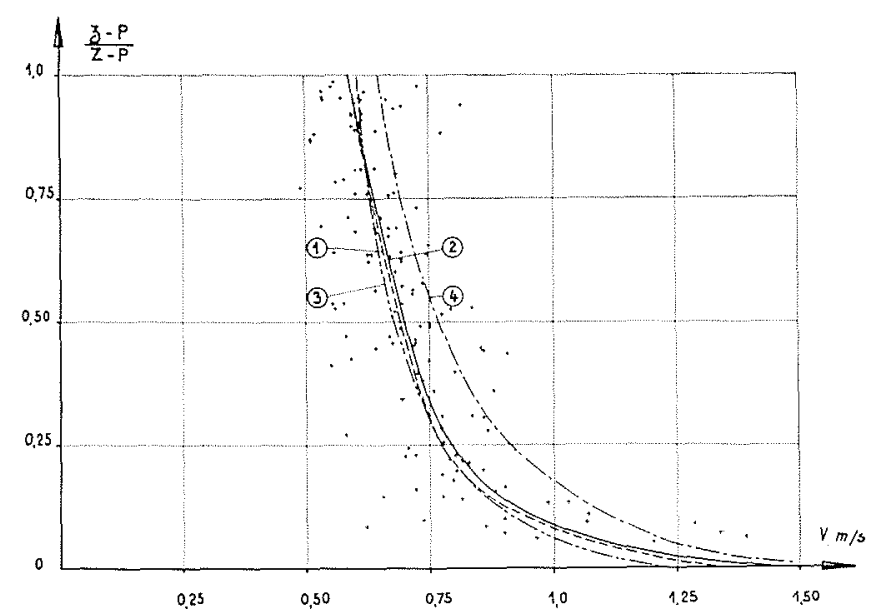

8/ 


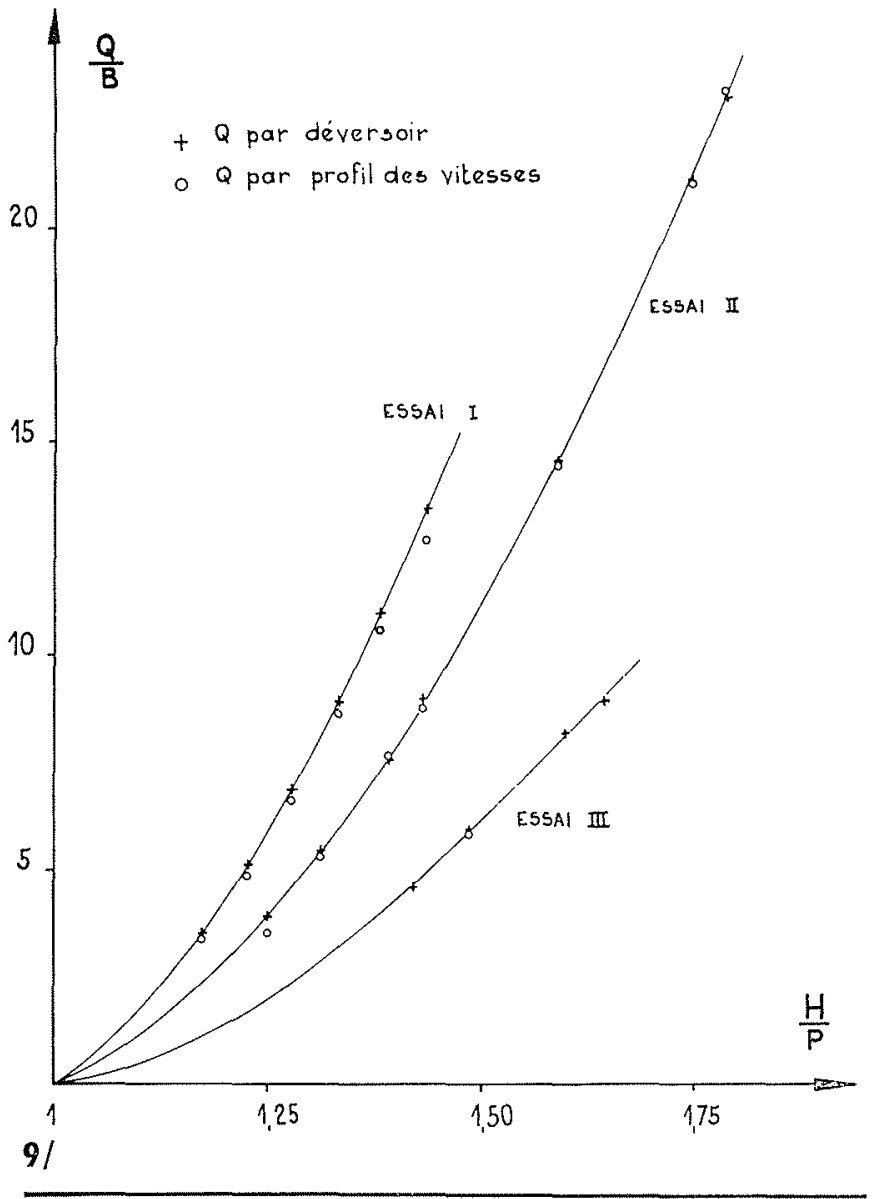

$(z-\mathrm{P})$, faite à partir des trois méthodes de mesures utilisées, conduit aux écarts suivants, $Q_{1}$ étant le débit de référence.

- profil $\overrightarrow{\mathrm{V}} \cos \tau$, essai III :

$$
\mathrm{Q}_{\mathrm{III}} / \mathrm{Q}_{\mathrm{B}}=1,7 \%
$$

- profil $\vec{V} \cos \tau$, essai IV:

$$
\mathrm{Q}_{\mathrm{IV}} / \mathrm{Q}_{\mathrm{I}}=1,8 \%
$$

- profil $\overrightarrow{\mathrm{V}} \cos \tau$, essai $\mathrm{V}$ :

$$
\mathrm{Q}_{\mathrm{V}} / \mathrm{Q}_{\mathrm{B}}=5,5 \%
$$

\section{Construction d'un abaque donnont les principoux paramètres inf́luençant l'écoulement}

\section{Généralités.}

La construction que nous proposons nécessite quelques hypothèses qui par suite s'avèreront être vérifiées:

- l'écoulement est plan (faible influence de l'angle $\alpha$ sur les vecteurs vitesse);

- le plan de charge est horizontal. (Les pertes de charge étant relativement faibles ont été négligées);

- l'énergie est constante sur toute la largeur du canal. Elle a été calculée dans le plan $\mathrm{S}_{2}$ où était mesuré $(\mathrm{H}-\mathrm{P})$.

On peut alors considérer que dans le plan $S_{1}$, la somme des énergies (đe pression, de position et cinétique) est égale à l'énergie calculée dans le plan $S_{2}$. De ce fait, toutes les pressions totales mesurées sont constantes.

Principe de la représentation en valeurs dimensionnelles.

Considérons deux axes rectangulaires dont les origines sont confondues avec l'arête du seuil, on porte en ordonnée $(z-\mathrm{P})$ et en abscisse les pressions exprimées en hauteur d'eau (fig. 10).

Les pressions totales mesurées se traduisent par une droite verticale d'abscisse $P_{t_{1}}=(E-P)$ [droite $(l)$ ]. L'écart maximal entre la moyenne des pressions totales et l'énergie calculée à l'amont est de $1,75 \%$. En retranchant de $(\mathrm{E}-\mathrm{P})$ la cote du point considéré $(z-P)$ on obtient une droite à $45^{\circ}$ coupant l'axe des ordonnées en $(E-P)$ [droite (2)] Nos dispositifs nous ont permis de mesurer les pressions statiques en tous points de la lame. Il a été facile de porter leur valeur :

$$
\mathrm{P}_{s}=p / \rho g+(z-\mathrm{P}) \quad \text { courbe }(3)
$$

ou encore:

$$
p / p g
$$

courbe (4).

L'intervalle entre $(I)$ et (3) ou (2) et (4) donne nécessairement le $\mathrm{V}_{1}^{2} / 2 \mathrm{~g}$ correspondant aux vitesses mesurées.

\section{Eîndue ce la représentation à tous les cas étudiés. Abaque adimentionnel.}

Une représentation adimensionnelle peut traduire tous les écoulements sur un seuil mince. Le praticien pourra donc indépendamment mesurer $(Z-P)$ en $S_{1}$ ou $(H-P)$ en $S_{2}$ et déduire de ce graphique les autres facteurs du déversement.

Nous avons choisi somme système de coordonnées

AXE DES ORDONNÉES.

Les différentes valeurs caractéristiques de $(z-P)$ ont été ramenées à $(Z-P)$ ainsi,

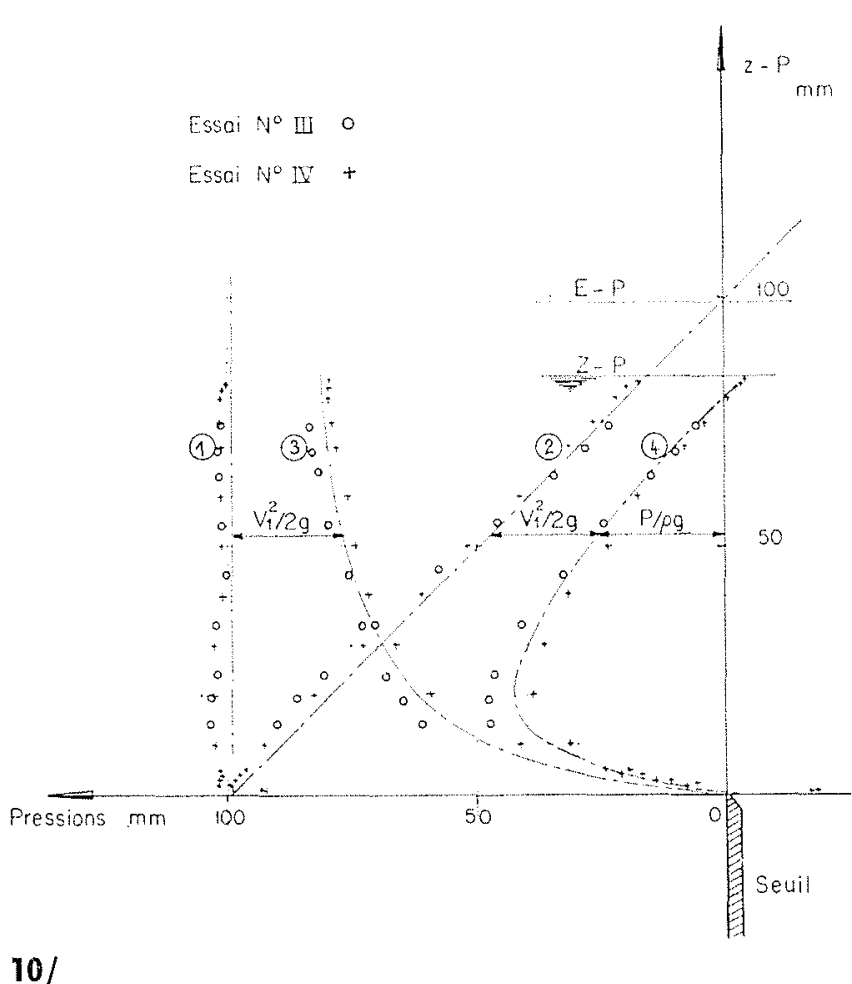


- pour $(z-P) /(Z-P)<1$ les points d'exploration sont compris dans la lame;

- pour $(z-P) /(Z-P)=1$ le point considéré est à la surface libre;

- enfin lorsque $(z-P) /(Z-P)>1$, on tend vers une limite qui est $(E-P) /(Z-P)$.

Nous avons pu vérifier dans la limite de nos expériences qu'il existait un rapport pratiquement constant entre $(E-P)$ et $(Z-P)$ soit comme l'indique la figure 11 :

$$
(E-P) /(Z-P)=1,2
$$

AXE DES ABSCISSES.

Les pressions totales et cinétiques sont diminuées de la pression de position et cela ramène au tirant d'eau en amont du seuil.

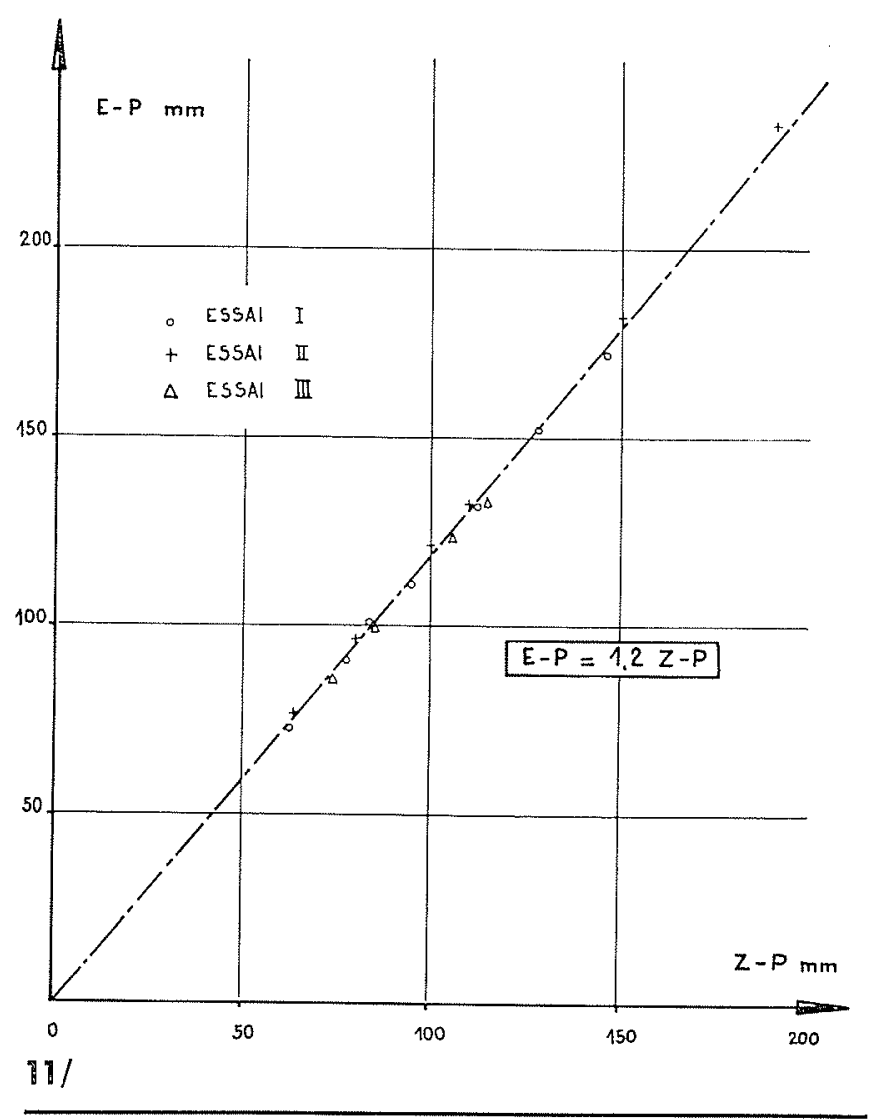

Pour une condition définie $(\mathrm{H}-\mathrm{P})$ on voit que lorsque $(z-P)=0$ le numérateur s'écrit $(E-P)$, et on définit en $S_{1}$ la valeur :

$$
\left(V^{2} / 2 g\right)+(p / \rho g)
$$

Le rapport est alors: $(\mathrm{E}-\mathrm{P}) /(\mathrm{H}-\mathrm{P})$.

Comme le terme $(p / \rho g)$ est nul en ce point, on a déterminé la vitesse à la partie inférieure de la lame.

Dans la même condition $(\mathrm{H}-\mathrm{P})$, lorsque :

$$
(z-\mathrm{P})=(\mathrm{Z}-\mathrm{P})
$$

on a au numérateur :

$$
(\mathrm{E}-\mathrm{P})-(\mathrm{Z}-\mathrm{P})=\mathrm{V}_{1}^{2} / 2 \mathrm{~g}
$$

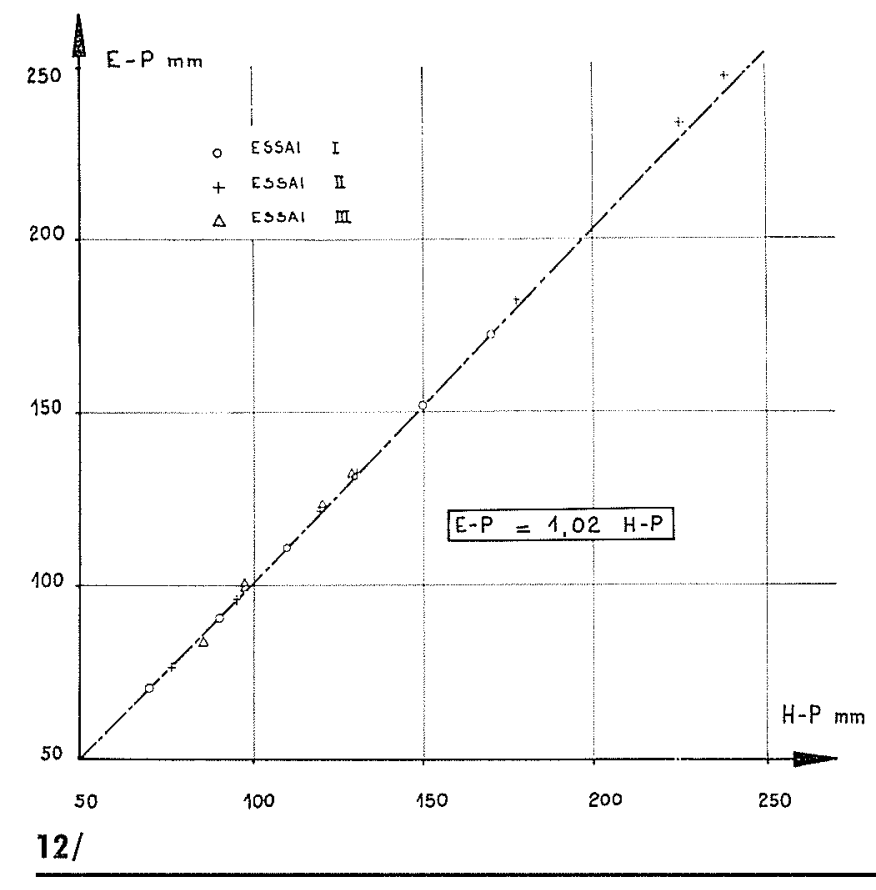

Le rapport s'écrit indépendamment :

$$
\frac{(E-P)-(Z-P)}{(H-P)}
$$

oll :

$$
\frac{\mathrm{V}_{1}^{2} / 2 g}{(\mathrm{H}-\mathrm{P})}
$$

On a défini de ce fait la vitesse à la surface libre.

Expérimentalement, nous avons obtenu un rapport constant entre $(\mathrm{E}-\mathrm{P})$ et $(\mathrm{H}-\mathrm{P})$ soit :

$$
(\mathrm{E}-\mathrm{P}) /(\mathrm{H}-\mathrm{P})=1,02
$$

EQUATION DE LA DROITE dE L'ÉNERGIE, RAMENÉE AUX PARAMÈTRES $(\mathrm{Z}-\mathrm{P})$ et $(\mathrm{H}-\mathrm{P})$.

La ligne d'énergie $(E-P)$ ramenée aux valeurs adimensionnelles donne une droite dont l'équation est :

$$
\frac{(\mathrm{E}-\mathrm{P})}{(\mathrm{Z}-\mathrm{P})}=-1,175\left(\frac{(\mathrm{E}-\mathrm{P})-(z-\mathrm{P})}{(\mathrm{H}-\mathrm{P})}\right)+1,2
$$

On peut constater que lorsque $(z-\mathrm{P}) /(\mathrm{Z}-\mathrm{P})$ prend les valeurs caractéristiques 0 et 1 , on établit un rapport constant entre la vitesse aux limites de la lame et le tirant d'eau en amont du seuil :

$$
\frac{\mathrm{V}_{1}^{2} / 2 g}{(\mathrm{H}-\mathrm{P})}
$$

Par contre lorsque $(z-\mathrm{P}) /(\mathrm{Z}-\mathrm{P})$ est compris entre ces deux limites, dans le rapport:

$$
\frac{(\mathrm{E}-\mathrm{P})-(z-\mathrm{P})}{(\mathrm{H}-\mathrm{P})}
$$

le numérateur s'exprime par l'énergie cinétique:

$$
\mathrm{V}^{2} / 2 g
$$

et l'énergie de pression ( $p / \rho g$ ) (fig. 13). 

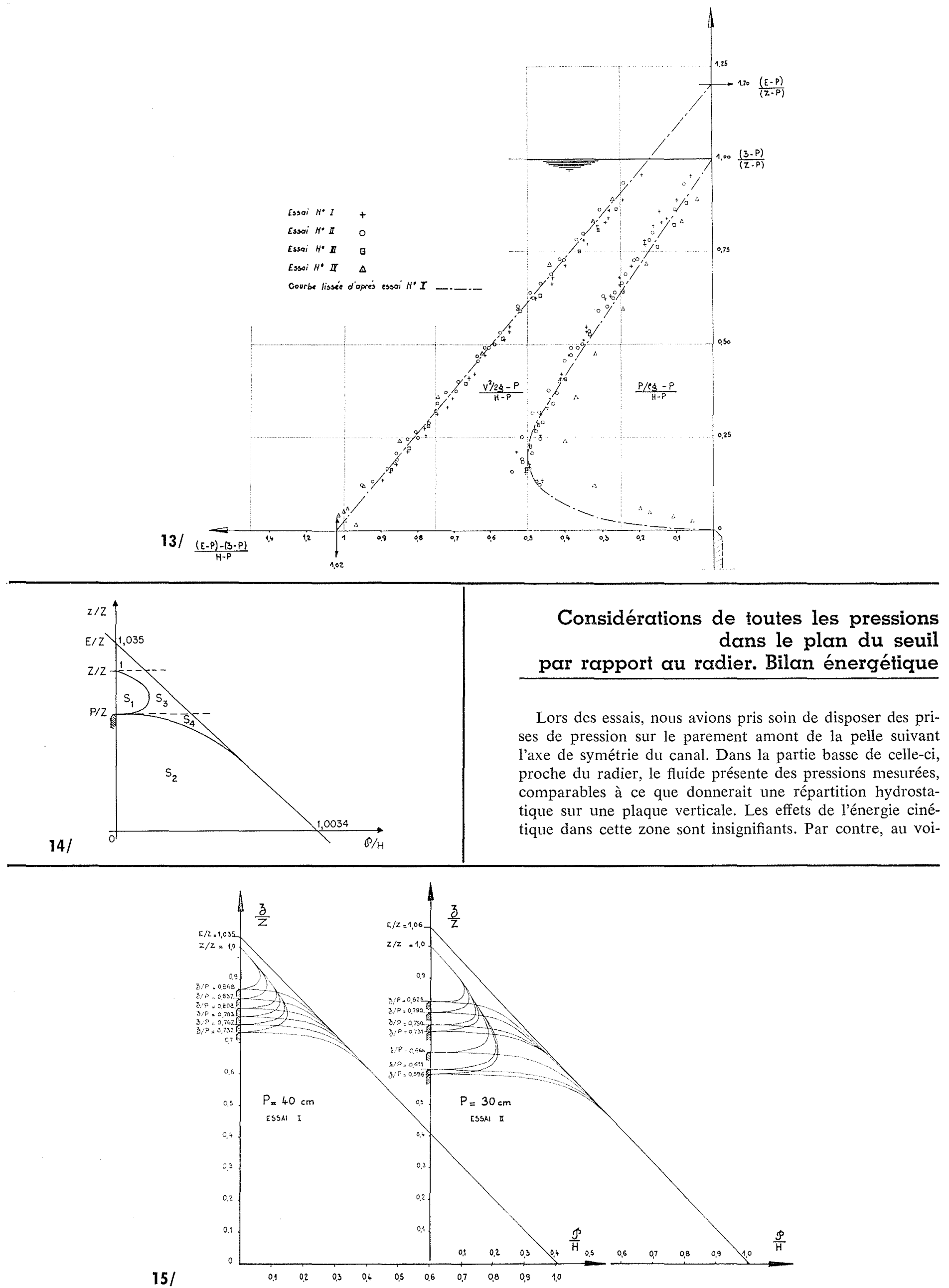
sinage de la crête la pression statique s'annule et l'on est dans la région où l'énergie cinétique est maximum.

\section{Représentation adimensionnelle des pressions.}

Dans la partie précédente de l'étude, nous avions considéré uniquement la lame déversante. Dans le cas présent, où tout est ramené au radier, il a été choisi pour l'axe des ordonnées le rapport $z / Z$ et pour l'axe des abscisses le rapport $\mathscr{T} / \mathrm{H}$.

L'étude de chacun de ces rapports montre:

a) Que pour l'axe des ordonnées, les valeurs caractéristiques seront les suivantes:

- lorsque $z=\mathrm{E}$ on aura un rapport $\mathrm{E} / \mathrm{Z}$. Dans tous les cas de l'essai $\mathrm{I}$, on a pu relever:

$$
1,021<\mathrm{E} / \mathrm{Z}<1,048
$$

finalement la moyenne a été adoptée et $\mathrm{E} / \mathrm{Z}=1,035$ avec une incertitude de $\pm 1,2 \%$. Dans tous les cas de l'essai II, on a pu relever:

$$
1,036<\mathrm{E} / \mathrm{Z}<1,088
$$

nous avons adopté $\mathrm{E} / \mathrm{Z}=1,061$ avec une incertitude de $\pm 1,7 \%$.

- lorsque $z=\mathrm{Z}$, le rapport est égal à l'unité.

- lorsque $z=\mathrm{P}$, le rapport vaut $\mathrm{P} / \mathrm{Z}$ et l'on obtient autant de points caractéristiques que l'essai a fait intervenir de tirants d'eau.

b) Que pour l'axe des abscisses nous avons choisi le rapport $\mathscr{T} / \mathrm{H}$ dans lequel $\mathscr{T}$ s'exprime par:

$$
(\mathrm{P}-\mathrm{z})+p / \rho g
$$

et devient maximum, c'est-à-dire égal à $E$ lorsque $z$ tend vers $\varepsilon$. En effet, il se trouve que $p / p g$ mesuré au manomètre représente exactement la valeur de $\mathrm{E}-\mathrm{P}$ pour cette condition, et $(\mathrm{E}-z)-(\mathrm{P}-z)$ dans le cas où $z \# 0$. Il est évident que lorsque $z$ tend vers $P$, la pression tend vers 0 .

Nous avons retenu comme valeur maximale de ce rapport:

- pour l'essai I : $\mathscr{P} / \mathrm{H}=1,0034$;

- pour l'essai II : $\mathscr{T} / \mathrm{H}=1,0075$.

La droite passant par $\mathrm{E} / \mathrm{Z}$ et par $\mathscr{T} / \mathrm{H}_{\max }$ est la ligne figurative de la pression totale en amont du déversoir dans le canal avant toute influence de la vitesse.

Ce mode de représentation permet de distinguer pour une même condition d'écoulement une courbe scindée en deux parties et dont le point de rebroussement se trouve à $P / Z$ (fig. 14). La partie supérieure comprise entre $Z / Z$ et $P / Z$ donne les pressions dans la lame, la partie inférieure comprise entre 0 et $P / Z$ donne les pressions sur le parement du seuil. Les surfaces désignées par $S_{1}$ et $S_{2}$ sont proportionnelles à l'énergie potentielle, les surfaces $S_{3}$ et $S_{4}$ sont proportionnelles à l'énergie cinétique.

On obtiendra donc autant de courbes que de conditions d'écoulement mais toutes aboutiront au point d'ordonnée $z / \mathrm{Z}=1$.

Les graphiques de la figure 15 donnent ce type de représentation pour les essais I et II. La différence des courbes est caractérisée uniquement par le point $P / Z$ qui décroît lorsque le tirant d'eau sur le seuil augmente.

\section{Bilan énergétique.}

Le graphique précédent présente une famille de courbes homologues, limitant des aires proportionnelles. Cha-

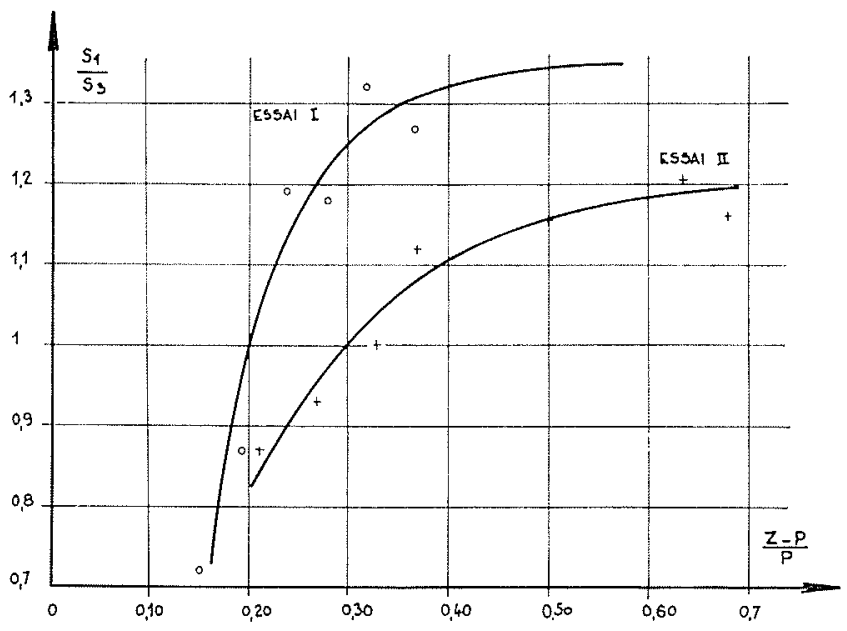

$16 /$

cune de ces aires représente une partie de l'énergie relative à l'écoulement, et la somme de $S_{1}, S_{2}, S_{3}$ et $S_{4}$ est bien égale à l'aire du triangle $0, \mathrm{E} / \mathrm{Z}, \mathscr{P} / \mathrm{H}$ qui n'est autre que la représentation de l'énergie totale.

En considérant deux zones distinctes, séparées par l'horizontale d'ordonnée $\mathrm{P} / \mathrm{Z}$ on voit que $S_{1}, S_{3}$ matérialise les pressions dans la lame au-dessus du seuil, $S_{2}, S_{4}$ les pressions sous la crête. Le planimétrage de toutes ces surfaces représentatives permet de constater les effets suivants:

En portant sur un graphique le rapport $S_{1} / S_{3}$ en fonction de $(Z-P) / P$ on obtient deux courbes distinctes propres à une hauteur de pelle.

A $\mathrm{P}$ constant lorsque $\mathrm{Z}-\mathrm{P}$ croit, le rapport $(\mathrm{Z}-\mathrm{P}) / \mathrm{P}$ croît également, les aires $S_{1}$ et $S_{3}$ sont nécessairement en augmentation, mais dans une certaine limite la pression statique croît plus vite que la pression relative à l'énergie cinétique.

On remarque sur la figure 16 que le rapport $S_{1} / S_{3}$ croît avec $(\mathrm{Z}-\mathrm{P}) / \mathrm{P}$ pour tendre vers une limite qui paraît être conditionnée par la hauteur de pelle.

Il nous a paru aussi intéressant de comparer l'énergie

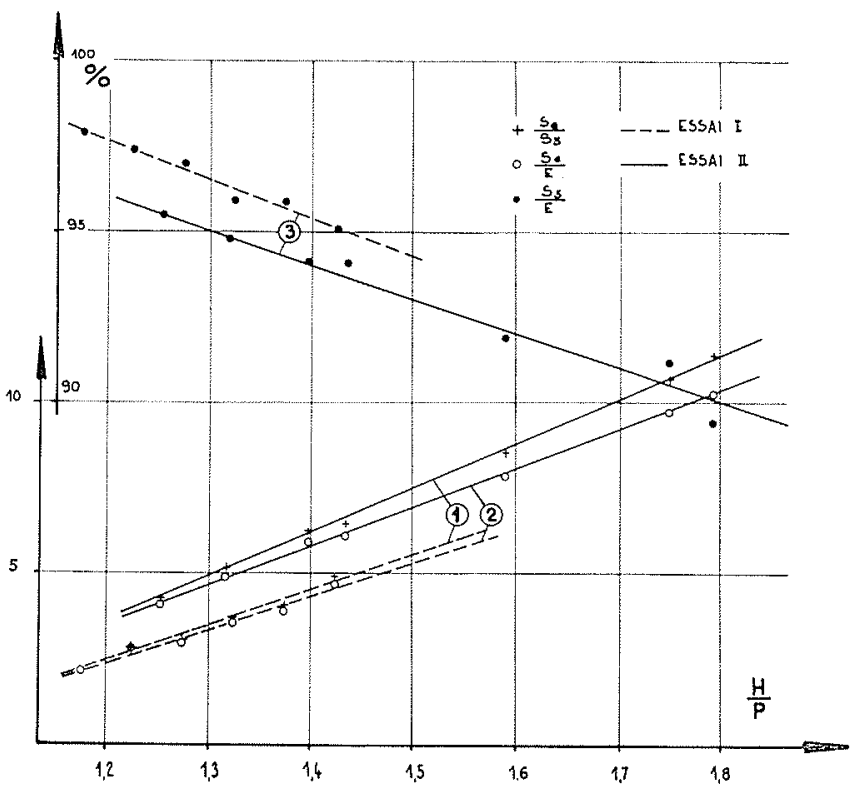

17/ 


\section{P. BOYER, L. CASTEX ef J. NOUGARO}

\begin{tabular}{|c|c|c|c|c|c|c|c|c|c|c|c|c|c|}
\hline \multicolumn{5}{|c|}{$P=30 \mathrm{~cm}$} & \multicolumn{9}{|c|}{ Aire totale $=213,2 \mathrm{~cm}^{2}=E$} \\
\hline$Z-P$ & $\frac{z-p}{p}$ & $s_{1}$ & $\mathrm{~S}_{2}$ & $\mathrm{~S}_{3}$ & $\mathrm{~S}_{4}$ & $\mathrm{~S}_{2}+\mathrm{S}_{4}$ & $\frac{s_{2}}{s_{2}+S_{4}}$ & $s_{1}+s_{3}$ & $\frac{s_{1}}{s_{1}+s_{3}}$ & $s_{1}+s_{2}$ & $\frac{S_{1}+S_{2}}{E}$ & $\mathrm{H} / \mathrm{P}$ & $s_{1} / s_{3}$ \\
\hline $\begin{array}{l}63,5 \\
80 \\
100 \\
110 \\
150 \\
191 \\
203\end{array}$ & $\begin{array}{l}0,212 \\
0,267 \\
0,333 \\
0,367 \\
0,500 \\
0,635 \\
0,678\end{array}$ & $\begin{array}{r}4,6 \\
6,4 \\
9,1 \\
11,2 \\
15,6 \\
20,7 \\
21,6\end{array}$ & $\begin{array}{l}199,1 \\
195,9 \\
191,6 \\
189,6 \\
180,4 \\
173,8 \\
169,1\end{array}$ & $\begin{array}{c}5,3 \\
6,9 \\
9,1 \\
10 \\
13,5 \\
17,2 \\
18,6\end{array}$ & $\begin{array}{l}3,4 \\
3,5 \\
3,5 \\
3 \\
3,2 \\
3,6 \\
3,2\end{array}$ & $\begin{array}{l}202,5 \\
199,4 \\
195,1 \\
19 \%, 6 \\
183,6 \\
177,4 \\
172,3\end{array}$ & $\begin{array}{l}0,983 \\
0,982 \\
0,982 \\
0,984 \\
0,982 \\
0,980 \\
0,981\end{array}$ & $\begin{array}{r}9,9 \\
13,3 \\
18,2 \\
21,2 \\
29,1 \\
37,9 \\
40,2\end{array}$ & $\begin{array}{l}0,464 \\
0,481 \\
0,500 \\
0,528 \\
0,536 \\
0,546 \\
0,537\end{array}$ & $\begin{array}{l}203,7 \\
202,3 \\
200,7 \\
200,8 \\
196,0 \\
194,5 \\
190,7\end{array}$ & $\begin{array}{l}0,955 \\
0,949 \\
0,941 \\
0,941 \\
0,919 \\
0,912 \\
0,894\end{array}$ & $\begin{array}{l}1,253 \\
1,317 \\
1,398 \\
1,435 \\
1,591 \\
1,751 \\
1,795\end{array}$ & $\begin{array}{l}0,870 \\
0,927 \\
1 \\
1,120 \\
1,155 \\
1,205 \\
1,160\end{array}$ \\
\hline \multicolumn{14}{|c|}{$P=40 \mathrm{~cm}$} \\
\hline $\begin{array}{c}60,3 \\
77,9 \\
95 \\
112,2 \\
128,2 \\
146,2\end{array}$ & $\begin{array}{l}0,151 \\
0,194 \\
0,238 \\
0,280 \\
0,320 \\
0,368\end{array}$ & $\begin{array}{l}2,3 \\
3,4 \\
5,5 \\
6,5 \\
8,5 \\
9,9\end{array}$ & $\begin{array}{l}200,4 \\
198,1 \\
195,2 \\
192,1 \\
190 \\
187\end{array}$ & $\begin{array}{l}3,2 \\
3,9 \\
4,6 \\
5,5 \\
6,4 \\
8,1\end{array}$ & $\begin{array}{l}1,3 \\
1,9 \\
1,5 \\
1,8 \\
1,6 \\
1,6\end{array}$ & $\begin{array}{l}201,7 \\
200 \\
196,7 \\
193,9 \\
191,6 \\
188,6\end{array}$ & $\begin{array}{l}0,993 \\
0,990 \\
0,992 \\
0,991 \\
0,991 \\
0,991\end{array}$ & $\begin{array}{l}5,5 \\
7,3 \\
10,1 \\
12 \\
14,9 \\
18\end{array}$ & $\begin{array}{l}0,418 \\
0,446 \\
0,544 \\
0,541 \\
0,570 \\
0,550\end{array}$ & $\begin{array}{l}202,7 \\
201,5 \\
200,7 \\
198,6 \\
198,5 \\
196,9\end{array}$ & $\begin{array}{l}0,979 \\
0,974 \\
0,970 \\
0,960 \\
0,959 \\
0,951\end{array}$ & $\begin{array}{l}1,175 \\
1,225 \\
1,275 \\
1,325 \\
1,375 \\
1,425\end{array}$ & $\begin{array}{l}0,720 \\
0,870 \\
1,190 \\
1,180 \\
1,320 \\
1,220\end{array}$ \\
\hline
\end{tabular}

cinétique totale $S_{6}=S_{3}+S_{4}$ à l'énergie des pressions statiques totales $S_{i}=S_{1}+S_{2}$, puis de les ramener à l'énergie totale E. En portant sur un graphique (fig. 17) les différents rapports $S_{6} / S_{5}, S_{5 /} / E, S_{6} / E$ exprimés en $\%$ en fonction de $\mathrm{H} / \mathrm{P}$ on peut voir l'évolution des transformations énergétiques.

- Le rapport $\mathrm{S}_{6} / \mathrm{S}_{5}$ [droite (l)] est traduit par une évolution linéaire lorsque $1<\mathrm{H} / \mathrm{P}<2$ et est influencé par la hauteur de pelle.

- Le rapport $S_{6} / E$ [droite (2)] donne en pourcent la transformation de l'énergie potentielle en énergie cinétique. L'accroissement de transformation est proportionnel au tirant d'eau, mais également conditionné par la hauteur de pelle.

- La surface $S_{\tilde{n}}$ représente ce qui demeure de l'énergie potentielle après transformation de l'énergie cinétique et n'est autre que l'énergie de pression statique. Le rapport $\mathrm{S}_{5} / \mathrm{E}$ donne en pourcent ce qui subsiste comme possibilités à l'écoulement [droite (3)].

- Pour une même valeur de $\mathrm{H} / \mathrm{P}$ et une même hauteur de pelle, on peut constater que la somme des rapports $\mathrm{S}_{6} / \mathrm{E}+\mathrm{S}_{5 / \mathrm{j}} / \mathrm{E}$ est bien égale à $100 \%$.

- De même pour une hauteur de pelle l'aire $S_{4}$ représentant l'énergie cinétique en dessous de la lame, semble demeurer constante quel que soit le tirant d'eau sur le seuil.

\section{Conclusions}

Le travail que nous venons de présenter est une suite logique aux résultats obtenus antérieurement. L'aboutissement de ces nombreuses mesures apportent une meilleure connaissance de la morphologie des lames déversantes.

Il y a quelques temps, notre Laboratoire publiait un article dans lequel il était proposé une relation donnant le coefficient de débit sur les déversoirs en mince paroi en fonction de l'énergie et de la hauteur de pelle.
L'énergie était déduite d'un calcul d'approximation, indispensable pour chaque condition d'écoulement. Nos relations donnent immédiatement cette grandeur, pour une mesure de $(\mathrm{H}-\mathrm{P})$ ou $(\mathrm{Z}-\mathrm{P})$.

D'autre part, la mesure de $(\mathrm{H}-\mathrm{P})$ qui doit être effectuée selon des normes impératives n'est pas souvent commode sur les déversoirs industriels. Il sera facile à l'hydraulicien de mesurer $(\mathrm{Z}-\mathrm{P})$ et d'apporter la correction proposée pour aboutir à $(\mathrm{H}-\mathrm{P})$ ou $(\mathrm{E}-\mathrm{P})$.

Enfin, notre construction permet, pour n'importe quel point de cote $z$ de connaître la vitesse ponctuelle, la pression statique. De plus, les vitesses aux limites de la lame étant parfaitement définies, il est possible d'intégrer avec précision leurs profils pour aboutir aux débits unitaires.

Bien que les conditions expérimentales de Bazin soient un peu différentes des nôtres, nous pouvons apprécier la bonne concordance des résultats et surtout la validité des hypothèses avancées.

Une étude plus approfondie en représentation adimensionnelle de toutes les pressions comptées à partir du radier du canal permettrait d'établir un bilan énergétique.

\section{Références bibliographiques}

Escande (L.), - Hydraulique générale.

Carlier (M.). - Cours d'hydraulique générale et machines hydrauliques.

LEnCAStre (A.). - Manuel d'hydraulique générale.

CRausse (E). - Hydraulique des canaux découverts en régime permanent.

BaziN (H.). - Expériences nouvelles sur l'écoulement en déversoir, Dunod (1968).

I.S.O. - Mesure de débits des liquides dans les canaux découverts au moyen de déversoirs à échancrures et canaux jaugeurs à ressaut.

Nougaro (J.), Barbe (A.), Coulomb (R.), Moreau de Saint-Martin et BOYer (P.). - Loi hauteur-débit d'un déversoir à nince paroi fonction de la charge totale et valable dans une large plage d'utilisation. La Houlle Blanche, $n^{\circ} 3$ (1967). 